\title{
Exploring wet markets
}

\author{
Wet markets have come under scrutiny amidst the COVID-19 pandemic. Calls to ban them may seem like a \\ straightforward component of preventing further pandemics, but controlling zoonotic infections requires a much \\ wider approach.
}

T here have been calls from the international political community and others in recent weeks for wet markets to be banned. In light of the COVID-19 pandemic, wet markets have been widely portrayed as hotspots for the transmission of disease from animals to humans - banning them seems like a pragmatic decision. There is, however, a rich and growing debate around targeting wet markets as an effective means of preventing future zoonotic disease with pandemic potential.

'Wet markets' is a collective term for heterogeneous businesses; the adjective 'wet' relates to the water and ice used to keep product and premises cool and clean. Most sell vegetables, meat, poultry or fish; some sell and slaughter live animals; some sell wildlife and exotic animals. But the moniker 'wet market' may have, in recent times, wrongly become synonymous for many with the capture and trade of wildlife, chaos and even criminality.

Wet markets support livelihoods, including those of small producers and farmers. They are an important source of food and nutrition for many communities and, importantly, a feature of many culinary cultures. The focus on 'freshness' from the wet market, and indeed the trusting relationship between vendor and consumer, is deeply valued in many societies (S. Zhong et al., Agric. Hum. Values 37, 175-185; 2020), though it is perhaps not the norm for societies more exposed to the ways of modern retail. The reported reduction in sales of live animals in wet markets over recent years, due to tightened regulations following previous zoonotic outbreaks, has been regarded as having a negative cultural impact. The scrutiny wet markets have come under due to the risk of zoonosis has eroded trust in this food source that is deeply embedded in many traditions and societies. It is argued that in the era of COVID-19, the operational and economic realities of wet markets have been misrepresented. The World Health Organization is currently advising that wet markets, when reopening, operate with much tighter terms of reference - particularly with respect to improved sanitation, improved regulation, the banning on sale and trade of wildlife for food, and stronger governance.

However, the calls to ban wet markets have been very strong and the argument that wet markets are prominent in the aetiology of zoonotic disease is persuasive, particularly with regard to their representation as enablers of capture, traffic, trade and slaughtering of wild, endangered and exotic animals. Recent portrayals of wet markets wild and domesticated species unnaturally coexisting and slaughtered side-by-side, in poor conditions of welfare and sanitation certainly support their position as hotspots of zoonosis. But, given the diversity of business that occurs in wet markets and the benefits that wet markets bring to society, culture, economy and health, that representation must be thoroughly explored and understood by the international community. Targeting activities in wet markets that facilitate zoonosis must be specific and explicit.

The COVID-19 pandemic has made food systems and zoonosis everyone's business. Food systems have social, economic, health and environmental even philosophical - domains; as does zoonosis, and so a technocratic approach to zoonosis through simply regulating or banning wet markets is inadequate. There are ample opportunities across the food supply chain for infectious agents to make the leap from animal to human populations. Numerous epidemics and pandemics were zoonotic in origin - HIV/AIDS, Ebola, Zika, the 2002-2004 outbreak of SARS, MERS and, indeed, zoonosis contributes to around $70 \%$ of human diseases. As the food system encroaches more on natural habitats, with intensification of food production and degradation of natural resources, biodiversity and wild habitats, wild animals and humans operate in closer proximity. They say that good fences make good neighbours - as we dismantle boundaries of land use and habitats for food production, how well do we know the neighbourhoods we are moving into?

Dialogue about zoonosis must extend far beyond wet markets. Zoonosis is a food systems issue. Joined-up thinking on food access, infrastructure, food security, food safety and sanitation, pest control, wild habitat biodiversity, and our relationship with animals must be addressed. This requires an interdisciplinary approach, and involves the interests of all sections of society.

Published online: 19 May 2020 https://doi.org/10.1038/s43016-020-0090-1 